\title{
Nurse educators' views on implementation and use of high-fidelity simulation in nursing programmes
}

\author{
E Powell, PhD, MA Cur Hons, BA Cur, BCur (Ed et Admin), RN, RM; B Scrooby, PhD, MCur, RN, RM, NE, NM; A van Graan, PhD, MCur, RN, RM, NE, NM
}

School of Nursing Science, Faculty of Health Sciences, North-West University, Potchefstroom, South Africa

Corresponding author: B Scrooby (belinda.scrooby@nwu.ac.za)

Background. Clinical skills development of student nurses is a concern in nursing education owing to limited clinical exposure and learning opportunities. High-fidelity simulation as a teaching-learning strategy creates an environment where student nurses develop clinical skills through interactive participation.

Objectives. The aim of this research study was to explore nurse educators' views of high-fidelity simulation as an educational approach in nursing programmes. Methods. A qualitative descriptive design was used. Data were collected through individual semi-structured interviews. Data saturation occurred within 19 interviews. The population consisted out of $33(N=33 ; n=19)$ nurse educators. Direct content analysis was done using Hsieh and Shannon's approach. The study was conducted at a South African private higher education institution.

Results. Five main themes were identified. The nurse educators had not been exposed to or had limited experience with high-fidelity simulation. Limited resources and/or the lack of nurse educators trained in high-fidelity simulation were identified. The nurse educators agreed that high-fidelity simulation would contribute to the enhancement of clinical skills development and theory and practice integration.

Conclusion. High-fidelity simulation is not implemented owing to limited equipment or experience in using the equipment optimally. The nurse educators see high-fidelity simulation as a solution and valuable training method where clinical skills are developed before the student nurse is exposed to the private clinical environment.

Afr J Health Professions Educ 2020;12(4):215-219. https://doi.org/10.7196/AJHPE.2020.v12i4.1411

Over the past 20 years, there has been a steady growth in the use of simulation as a clinical teaching-learning strategy in nursing education. ${ }^{[1]}$ Simulation using high-fidelity manikins is used to teach student nurses skills that are necessary to reduce the frequency and harm attributable to errors in the healthcare setting. ${ }^{[1,2]}$ Nurse educators need to use high-fidelity simulation (HFS) to enhance clinical skills development in student nurses due to limited clinical exposure, difficulty in clinical placements and decreasing learning opportunities during work-integrated learning, especially as experienced in the private healthcare environment. ${ }^{[2,3]}$ Simulation training encourages the student nurse to use higher-order thinking skills while in a safe environment, to make sense of and to integrate new experience into the schema that they are constructing of 'how things are'. This simulation environment mimics the real world with real patient problems where students can better integrate theory and practice principles. ${ }^{[4]}$ Berragan $^{[3]}$ emphasises that simulation training creates an environment where collaboration and participation take place, while clinical skills are developed and students are prepared for the real world of nursing.

Successful implementation of HFS depends on the support of nurse educators. ${ }^{[5]}$ Paige and Morin ${ }^{[6]}$ state that there are only a few studies that have explored nurse educators' assumptions and beliefs on HFS as teachinglearning strategy, and that nurse educators hold varying beliefs regarding this phenomenon due to their views, perspectives, cultural differences and the availability of resources.

In order to fill the gap in the knowledge base in the field of nursing education, the main objective of this research is to explore and describe nurse educators' views of HFS as an educational approach in the nursing programmes of a South African private higher education institution (SAPHEI).

\section{Methods}

A qualitative descriptive research design was used. ${ }^{[7]}$ The population consisted of $33(N=33)$ nurse educators at a SAPHEI. A randomised purposive sampling method was used. The inclusion criteria were: head nurse educators and nurse educators permanently employed at one of six learning centres at the SAPHEI (the remaining learning centre where the researcher is employed as the manager was excluded from the study); learning and development facilitators and clinical facilitators permanently employed at the clinical facilities affiliated with the SAPHEI; and participants who took part in the quantitative phase of the research study and each voluntarily consented to take part in an individual semi-structured interview. The researcher selected a representative sample from each of the six learning centres of the SAPHEI. Data were collected using individual, semi-structured interviews of approximately 45 minutes each. ${ }^{[8]}$ Data saturation was obtained as no new concepts emerged after 19 interviews. The interview guide consisted of two parts, including firstly an introduction that read:

'As nurse educators, our goal is to improve clinical competence of student nurses through the use of various teaching methods. HFS is one of those teaching methods used to enhance clinical competence.' The following part included five open-ended questions: (i) What are your views about the implementation of HFS as a teaching method in nursing programmes at your institution?; (ii) What are your expectations of HFS as a teaching method?; (iii) What are your perceptions of HFS as a teaching method to enhance clinical competence of nursing students?; (iv) What is your opinion about the current practices and use of HFS in your institution?; and $(v)$ What value does HFS add as a teaching method in a nursing programme? 
Direct content analysis was done using Hsieh and Shannon's ${ }^{[9]}$ approach, identifying five themes. The variables identified during the quantitative phase of the research study were used together with key concepts identified during the qualitative phase of the research as initial coding categories. Transcripts were read and all text that on first impression appeared to present identified themes was highlighted. Coding of highlighted passages was done using the predetermined codes. Data that could not be placed in the predetermined codes were analysed to determine if they represent a new category or subcategory on an existing code. A co-coder was used to determine the accuracy of the research findings.

\section{Ethical considerations}

Ethical approval for the study was obtained from the University Health Research Ethics Committee and the SAPHEI. The North-West University
Health Research Ethics Committee (ref. no. NWU-00011-18-A1) provided written consent to the researcher to conduct this research. The participants gave written informed consent to participate in this study and could withdraw at any time. Interviews were recorded, transcribed and saved on a password-protected external hard drive. Confidentiality was maintained by coding of data to ensure anonymity.

\section{Results}

The following main themes, themes and sub-themes were identified, as outlined in Table 1 .

\section{Main theme 1: Experiences using HFS}

Under this main theme, two themes named 'no or very few experiences' and 'use only low-fidelity simulation' with their respective sub-themes emerged.

Table 1. Main themes identified from the views of nurse educators on implementing and use of HFS in nursing programmes

\begin{tabular}{|c|c|c|}
\hline Main themes & Themes & Sub-themes \\
\hline \multirow[t]{5}{*}{ Experiences using HFS } & \multirow[t]{3}{*}{ No or very few experiences } & Not actively involved in simulation training \\
\hline & & Have very little exposure \\
\hline & & Do not use it at all \\
\hline & \multirow{2}{*}{ Use only low-fidelity simulation } & Do not have high-fidelity manikins \\
\hline & & Experiences with low-fidelity simulation only \\
\hline \multirow[t]{7}{*}{ Comfort using HFS } & \multirow[t]{4}{*}{ Equipment available for simulation } & Do not have equipment \\
\hline & & Only have low-fidelity simulation equipment \\
\hline & & Budget constraints \\
\hline & & Too expensive \\
\hline & \multirow[t]{3}{*}{ Training received to use HFS } & Not enough training \\
\hline & & Need follow-up training \\
\hline & & Need trained facilitator to assist \\
\hline \multirow[t]{6}{*}{ Learning environment } & \multirow[t]{3}{*}{ Simulation creates a safe environment for students to practise } & Students experience less stress \\
\hline & & Patient is safe from inexperienced learners \\
\hline & & Invasive procedures are practised in safe environment \\
\hline & \multirow[t]{3}{*}{ Scenarios used in simulation must represent the clinical setting } & Realistic clinical setting/scenarios \\
\hline & & Controlled environment with planned outcomes \\
\hline & & Safe time/no interruptions \\
\hline \multirow[t]{14}{*}{ Skill development } & \multirow[t]{8}{*}{ Development of psychomotor skills } & Demonstrations of skills \\
\hline & & Not all skills are found at the patient's beds \\
\hline & & Set goals to improve skills \\
\hline & & Should not replace clinical practice \\
\hline & & Competent before going to patient \\
\hline & & Assess competency \\
\hline & & More approachable to attempt tasks \\
\hline & & Refresh educator's skill \\
\hline & \multirow[t]{6}{*}{ Development of cognitive and affective skills } & Develop clinical reasoning/judgement \\
\hline & & Effective communication \\
\hline & & Share ideas \\
\hline & & Encourage students to interact \\
\hline & & Build student-facilitator report \\
\hline & & Must not replace communication with the patient \\
\hline \multirow[t]{3}{*}{ Theory-practice integration } & \multirow[t]{3}{*}{ Theory integrated in practice } & Blended learning \\
\hline & & Understand theory better because it is visual \\
\hline & & Audiovisual stimulation \\
\hline
\end{tabular}




\section{No or very few experiences with HFS}

Sub-themes identified were: 'not actively involved in simulation training'; 'have very little exposure'; and 'do not use it at all'.

Al-Ghareeb and Cooper ${ }^{[10]}$ state that HFS as a teaching tool is undervalued and underused. The challenge using HFS is the limited resources and knowledge available on how to use it, as HFS training is demanding technological resources. ${ }^{[1]]}$ In supporting the literature, the participants stated:

'I have limited experience.' (participant 2; age 59 years; experience 12 years)

'We do not have the exposure or the means to train on that equipment.' (participant 4; age 42 years; experience 9 years)

'I just feel we are underutilising them.' (participant 18; age 42 years; experience 9 years)

\section{Use only low-fidelity simulation}

Sub-themes identified were: 'do not have high-fidelity manikins'; and 'experiences with low-fidelity simulation only'.

Low-fidelity simulation creates a semblance of reality by using part-task trainers or static manikins with props and techniques such as role play ${ }^{[12]}$ Lowfidelity simulation is preferred owing to the perception of nurse educators that the implementation of HFS is difficult and requires extensive content to development simulations for the classroom environment. ${ }^{[13]}$ In supporting the literature, the participants stated:

'We have a manikin in the simlab. It is really basic.' (participant 4; age 42 years; experience 9 years)

'We have a low-fidelity doll.' (participant 10; age 36 years; experience 1 year).

'I will say low-fidelity in terms of it is only the arm.' (participant 14; age 46 years; experience 16 years)

\section{Main theme 2: Comfort using HFS}

Under this main theme, two themes named equipment available for simulation' and 'training received to use HFS', with their respective subthemes, emerged.

\section{Equipment available for simulation}

Sub-themes identified were: 'do not have equipment'; 'only have low-fidelity simulation equipment'; 'budget constraints'; and 'too expensive'.

Achieving high-quality simulation experiences requires clear learning objectives to articulate the curriculum, and sufficient high-quality simulation resources, including adequately prepared staff. ${ }^{[14]}$ Equipment needed for HFS includes full-bodied manikins with advanced technology and an environment that resembles a particular healthcare setting. ${ }^{[15]}$ It is costly to set up HFS as the equipment and its maintenance is expensive. ${ }^{[16]}$ In supporting the literature, participants stated:

'If I have larger groups of students, how many educators do you have and how many simulation dolls do you have?' (participant 7; age 53 years; experience 15 years)

'We did put it on the budget but it was so expensive to have the right equipment available.' (participant 12; age 48 years; experience 17 years)

'There must be enough equipment and IT support on the premises because it can happen at any time that the programme has power outage. (participant 13; age 62 years; experience 30 years)
'There is a very big financial layout with putting together a decent simlab.' (participant 17; age 51 years; experience 8 years)

\section{Training received to use HFS}

Sub-themes identified were: 'not enough training'; 'need follow-up training'; and 'need trained facilitator to assist'.

HFS places demands on the technological ability of nurse educators because it necessitates greater engagement than other passive forms of instruction. ${ }^{[10]}$ To implement HFS as a teaching-learning strategy in a nursing curriculum, nurse educators need to be equipped with the necessary simulation-based teaching skills through workshops and skills training. ${ }^{[15]}$ In supporting the literature, the participants stated:

'I think one of the things we must look at is upskilling of the educators. I just did not have time to really go and learn how to use the equipment.' (participant 3; age 41 years; experience 10 years)

'The educator needs to be well trained.' (participant 10; age 36 years; experience 1 year)

'Your educators have to be taught how to work with the equipment.' (participant 13; age 62 years; experience 30 years)

\section{Main theme 3: Learning environment}

Under this main theme, two themes named simulation creates a safe environment for students to practise' and 'scenarios used in simulation must represent the clinical setting, with their respective sub-themes, emerged.

\section{Simulation creates a safe environment for students to practise}

Sub-themes identified were: 'student experiences less stress'; 'patient is safe from inexperienced learners'; and 'invasive procedures are practised in safe environment before attempting it on a patient'.

Nurse educators are challenged with providing an engaging and motivating learning environment for clinical nursing education. ${ }^{[17]}$ Simulation provides an opportunity for student nurses to acquire experience in a safe environment by allowing them to make mistakes without causing harm to the patient. ${ }^{[18]}$ In supporting the literature, the participants stated:

'Simulation is a safe area for students. It develops their skills before they practise this on a patient.' (participant 3; age 41 years; experience 10 years)

'In a sense give safety to the student for her to experiment and just get over her initial fear.' (participant 7; age 53 years; experience 15 years) 'A safe environment, where my student can practise without feeling that they are putting a patient at risk. An environment where there is no judgement.' (participant 11; age 58 years; experience 9 years)

\section{Scenarios used in simulation must represent the clinical setting}

Sub-themes identified were: 'realistic clinical setting/scenarios'; 'controlled environment with planned outcomes'; and 'safe time/no interruptions'.

Student nurses gain confidence before providing patient care in clinical practice through repeating practices in a more realistic learning environment. ${ }^{[18]}$ Nurse educators should be skilled practitioners in order to integrate simulation appropriately into nursing programmes, and should not merely have knowledge of learning theories and how to integrate simulation into the curriculum, but should also demonstrate practical expertise and expert knowledge of the subject matter. ${ }^{[19]}$ In supporting the literature, the participants stated: 
'It will enhance the whole learning and training setup ... it reflects the real thing.' (participant 1; age 57 years; experience 6 years)

'Students view simulation as a much more appropriate way of learning skills before going to the actual clinical environment. Not all the procedures are available in the clinical environment.' (participant 3; age 41 years; experience 10 years)

'The better the simulation environment, the more accurate and competent the results will be for your student. Educators to know how to properly facilitate the high-fidelity simulation to ensure that we use it comprehensively to enable the students.' (participant 9; age 48 years; experience 5 years)

\section{Main theme 4: Skills development}

Under this main theme, two themes named 'development of psychomotor skills' and 'development of cognitive and affective skills', with their respective sub-themes, emerged.

\section{Development of psychomotor skills}

Sub-themes identified were: 'demonstrations of skills'; 'not all skills are found at the patient's beds'; 'set goals to improve skills'; 'should not replace clinical practice'; 'competent before going to patient'; 'assess competency'; 'more approachable to attempt tasks'; and 'refresh educator's skill'.

Clinical experiences often focus on tasks or skill performance, which is problematic to find owing to a complex healthcare system and difficulty in finding clinical placements for student nurses. ${ }^{[20]}$ Therefore HFS has been suggested as an alternative to face-to-face clinical experience. Simulation allows student nurses to improve their cognitive, affective and psychomotor skills and make fewer medical errors, thus contributing to the protection of patients. ${ }^{[18]}$ In supporting the literature, the participants stated:

'They will be hands-on after completion of their training.' (participant 1; age 57 years; experience 6 years)

'Fill the gap where our students do not have the opportunity to either nurse patients with a particular condition or just because the patients are not being keen on being guinea pigs.' (participant 2; age 59 years; experience 12 years)

\section{Development of cognitive and affective skills}

Sub-themes identified were: 'develop clinical reasoning/judgement'; 'effective communication'; 'share ideas'; 'encourage students to interact'; 'build studentfacilitator report'; and 'must not replace communication with the patient'.

Student nurses are expected to gain knowledge, critical thinking and psychomotor skills that will enable them to make clinical judgements about care and manage complex clinical situations. ${ }^{[10]}$ In supporting the literature, the participants stated:

'We know [that] by doing, people develop more knowledge or gain more knowledge.' (participant 6; age 59 years; experience 29 years)

'Set different type of scenarios for a student, so that you can test their knowledge. I think it can also assist in theoretical training, because now you get an enforcement of what you discussed maybe in a theoretical facilitation session.' (participant 15; age 54 years; experience 13 years)

The healthcare needs of a patient must always take priority over the education needs of the student nurse. However, in simulation the student's needs are placed at the centre of attention. ${ }^{[3]}$ HFS can improve student nurses' current knowledge, skill performance, clinical judgement and affective skills if they receive the correct support, including: a facilitator in the simulation room; supportive feedback; role modelling of expected performance; an opportunity to repeat their performance; and communication tools. ${ }^{[21]}$ In supporting the literature, the participants stated:

'It will be difficult for a student to be able to empathise with the patient and reflect on what the patient is feeling when the patient is a manikin. (participant 3; age 41 years; experience 10 years)

'Other students feel alienated from this doll and struggle to see it as a human being that can react and they know it's me speaking. So they constantly ignore the doll and speak to me.' (participant 7; age 53 years; experience 15 years)

\section{Main theme 5: Theory-practice integration}

Under this main theme, one theme named 'theory is integrated in practice' with its respective sub-themes emerged.

\section{Theory is integrated in practice}

Sub-themes identified were: 'blended learning'; 'understand theory better because it is visual'; and 'audio-visual stimulation'

Simulation provides a safe environment where student nurses can experience different learning strategies that will allow them to integrate their knowledge and apply it to real patients. ${ }^{[18]}$ Problem-based learning will prepare student nurses for clinical practice by letting them solve problems during their clinical skills training, allowing them to integrate theory into practice. ${ }^{[17]}$ High-fidelity simulation-based learning can facilitate the acquisition of clinical reasoning skills, because the student nurse reflects on her/his actions and discusses alternative actions. ${ }^{[18]}$ The integration of an interactive learning method such as HFS in a nursing programme is important as it results in the development of more qualified and skilled nursing practitioners. ${ }^{[18]}$ In supporting the literature, the participants stated:

'It will create opportunities for students to develop their clinical skills as well as their critical thinking, clinical judgement. When you create a scenario you are allowing them to make decisions. So part of that decision-making process will end up in clinical judgement.' (participant 3 ; age 41 years; experience 10 years)

'You can combine your theoretical explanation with a practical demonstration. So it assists with theory, theory to the practice.' (participant 7; age 53 years; experience 15 years)

'They use all types of learning as they go around. To collaborate all of that together, and having that visual and auditory together with the aesthetic.' (participant 9; age 48 years; experience 5 years)

'It will help with the development of clinical judgement. It will help them with decision-making. With problem solving, they have to think, but again I have to give them a proper scenario.' (participant 10; age 36 years; experience 1 year)

'I think you can set up simulation scenarios, to see how their theorypractice integration is.' (participant 15; age 54 years; experience 13 years)

'It makes a lot more sense when you can actually visually see what the person is talking about. Be able to practise and I think more collaborative learning can take place. They can share their experiences a lot more.' (participant 16; age 44 years; experience 4 years) 


\section{Discussion}

Nurse educators at the SAPHEI have not been exposed or have limited experience using HFS as a teaching strategy. Venkatasalu et al. ${ }^{[22]}$ concur that simulation-based training is not yet widely used, as its effectiveness in nursing schools is not understood. The equipment currently in use in the majority of the clinical settings of the SAPHEI is low-fidelity manikins. The equipment and facility for HFS is not always available or used optimally owing to cost and availability of space to create a simulation environment effective enough to accommodate bigger classes. Nurse educators' lack of training and/or the lack of technological skills are the cause of their 'fear of the unknown' and resistance to using HFS. Nurse educators expressed the need for technical support from experts, and more available time in their programme to develop and plan simulation scenarios.

HFS is seen as a valuable training method in a safe environment where clinical skills are developed before the student nurse is exposed to the clinical environment. It enhances student nurses' confidence and competence, leading to safe patient care. ${ }^{[18]}$ Nurse educators need to be hands-on and knowledgeable about HFS as a teaching method, demonstrating practical expertise and expert knowledge of the subject matter in order to assist student nurses to obtain the necessary psychomotor and cognitive skills in a realistic environment that represents clinical practice. It cannot, however, replace real clinical practice. Affective skills should be learned and developed in the clinical setting through interaction with a patient.

HFS makes use of teaching-learning methods via which student nurses can enhance their clinical judgement and problem-solving skills by engaging with a dynamic learning environment. ${ }^{[17]}$ It integrates theory into practice through a hands-on approach where student nurses exercise solving patient problems during HFS case studies that empower the student to integrate their theoretical knowledge into clinical practice by solving problems using their reasoning skills as well as reflection on nursing care done.

\section{Study limitation}

The research study was conducted at a single SAPHEI, therefore the findings of this study can only be used as guide for other SAPHEIs.

\section{Recommendations}

The recommendations for research, education and practice are given below:

- develop a practice model to implement HFS in the nursing programmes at a SAPHEI;

- HFS as a clinical teaching-learning method should be added to the curriculum of the nursing programmes presented at the SAPHEI; and

- nurse educators and student nurses should be trained to use HFS as a clinical teaching-learning method to enhance clinical skills development through theory-practice integration, which will lead to better patient safety.

\section{Conclusion}

Simulation training encourages the student to think innovatively using higher-order thinking skills to make sense of and enable students to integrate each new experience while in a safe environment. This valuable teaching method can assist student nurses to develop their critical thinking skills, which would become evident in student nurses' ability to assess, identify and plan nursing action according to the patient's needs and diagnoses, and assist to immediately identify any abnormality presented in the patient, acting promptly in order to prevent complications by implementing meaningful actions.

\section{Declaration. None.}

Acknowledgements. We acknowledge financial and material support for language editing and publication fees from our affiliated university.

Author contributions. The first author collected the data and analysed it as well as wrote a chapter on qualitative data as part of her $\mathrm{PhD}$ thesis. The first author also wrote this article according to the journal's guidelines. The second and third authors as the first author's promotor and co-promotor reviewed and revised the article. The second author submitted the article to the journal. All authors revised the article and the second author finalised it.

Funding. This project was self-funded. North-West University gave financial support in the form of a postgraduate bursary.

Conflicts of interest. None.

1. Garden AL, Le Fevre DM, Waddington HL, Weller JM. Debriefing after simulation-based non-technical skill training in healthcare: A systematic review of effective practice. Anaesth Intensive Care 2015;43(3):300-308. https://doi.org/10.1177/0310057x1504300303

2. Garrett BM, MacPhee M, Jackson C. Implementing high-fidelity simulation in Canada: Reflections on 3 years of practice. Nurse Educ Today 2011;31(2011):671-676. https://doi.org/10.1016/j.nedt.2010.10.028

3. Berragan L. Simulation: An effective pedagogical approach for nursing? Nurse Edu Today 2011;31(2011):660-663 https://doi.org/10.1016/j.nedt.2011.01.019

4. Bambi D, Washburn J, Perkins R. Outcomes of clinical simulation for novice nursing students: Communication, confidence, clinical judgment. Nursing Educ Res 2009;30(2):79-82.

5. Jones AL. Faculty members' comfort levels in utilizing high-fidelity simulation in a Bachelor of Science nursing programme. PhD thesis. Vermillion: University of South Dakota, 2005.

6. Paige JB, Morin KH. Using Q-methodology to reveal nurse educators' perspectives about simulation design. Clinical Sim Nursing 2015;11(1):11-19. https://doi.org/10.1016/j.ecns.2014.09.010

7. Mouton J, Marais HC. Basic Concepts in the Methodology of the Social Sciences. Pretoria: Human Sciences Restorch J, Marais HC. Bancil 1996.

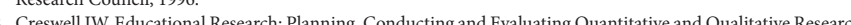
4th ed. Edinburgh: Pearson, 2014.

4. Hsieh H, Shannon SE. Three approaches to qualitative content analysis. Qual Health Res 2005;15(9):1277-1288.
. Hsieh H, Shannon SE. Three approaches to qual/t
https://doi.org/10.1177\%2F1049732305276687

10. Al-Ghareeb AZ, Cooper SJ. Barriers and enablers to the use of high-fidelity patient simulation manikins in nurse education: An integrative review. Nurse Edu Today 2016;36(2016):281-286. https://doi.org/10.1016/j. nedt.2015.08.005

11. Walker DM, Holme F, Zelek ST, et al. A process evaluation of PRONTO simulation training for obstetric and neonatal emergency response teams in Guatemala. Medical Edu 2015;15(117):1-8. https://doi.org/10.1186 s12909-015-0401-7

12. Winkelmann ZK, Eberman LE, Edler JR, Livingston LB, Games KE. Curation of a simulation experience by the clinical scholar: An educational technique in postprofessional athletic training. Athletic Train Educ J 2018;13(2):185-193. https://doi.org/10.4085/1302185

13. Sharpnack PA, Madigan EA. Using LFS with sophomore nursing students in a Baccalaureate nursing program. Nurs Edu Perspect 2012;33(4):264-268. https://doi.org/10.5480/1536-5026-33.4.264

14. Forber J, DiGiacomo M, Davidson P, Carter B, Jackson D. The context, influences and challenges for undergraduate nurse clinical education: Continuing the dialogue. Nurse Edu Today 2015;35(2015):1114-1118. https://doi.org/10.1016/j.nedt.2015.07.006

15. Welman A, Spies C. High-fidelity simulation in nursing education: Considerations for meaningful learning Trends Nursing 2016;3(1):1-16. https://doi.org/10.14804/3-1-42

6. Weller J, Cumin D, Torrie J, et al. Multidisciplinary operating room simulation-based team training to reduce treatment errors: A feasibility study in New Zealand hospitals. N Z Med J 2015;128(1418):40-51.

17. Koivisto JM, Haawisto E, Niemi H, Hano P, Nylund S, Multisilta J. Design principles for simulation games for learning clinical reasoning: A design-based research approach. Nurse Edu Today 2018;60(2018):114-120. https:/ doi.org/10.1016/j.nedt.2017.10.002

8. Eyikara E, Baykara ZG. Effect of simulation on the ability of first year nursing students to learn vital signs. Nurse Edu Today 2018;60(2018):101-106. https://doi.org/10.1016/j.nedt.2017.09.023

19. Topping A, Bøje RB, Rekola L, et al. Towards identifying nurse educators' competencies required for simulationbased learning: A systemised rapid review and synthesis. Nurse Edu Today 2015;35(2015):1108-1113. https://doi org/10.1016/.jnedt.2015.06.003

20. Zapko KA, Ferranto MLG, Blasiman R, Shelestak D. Evaluating best educational practices, student satisfaction, and self-confidence in simulation: A descriptive study. Nurse Edu Today 2018;60(2018):28-34. https://doi. and self-confidence in simulation:

21. Erlam G, Smythe L, Wright-St Clair V. Action research and millennials: Improving pedagogical approaches to Erlam G, Smythe L, Wright-St Clair V. Action research and millennials: Improving pedagogical approaches to
encourage critical thinking. Nurse Edu Today 2018;61(2018):140-145. https://doi.org/10.1016/j.nedt.2017.11.023 22. Venkatasalu MR, Kelleher M, Shao CH. Reported clinical outcomes of HFS versus classroom-based end-of-life care education. Int J Palliative Nurs 2015;21(4):179-186.

Accepted 10 September 2020 